\title{
Expression of bone matrix protein (Osteopontin) in dentogingival junction in periodontal disease
}

\author{
Olfat Mohamed Gab Allah, Mona Assem Abd-Elmotelb, Reda Gaber Saleh
}

Oral Biology Department, Faculty of Dentistry, Tanta University, Tanta, Egypt

\section{Email address:}

omaeg_2000@yahoo.com (O. M. Gab Allah), mon_assem@hotmail.com (M. Abd-Elmotelb), redag2000@gmail.com (R. G. Saleh)

\section{To cite this article:}

Olfat Mohamed Gab Alla, Mona Assem Abd-Elmotelb, Reda Gaber Saleh. Expression of Bone Matrix Protein (Osteopontin) in Dentogingival Junction in Periodontal Disease. American Journal of Life Sciences. Vol. 2, No. 6, 2014, pp. 400-405.

doi: 10.11648/j.ajls.20140206.21

\begin{abstract}
Recent breakthrough studies gave a considerable interest on the pathogenesis of periodontal diseases, particularly, the critical role of cytokines. Cytokines have dual roles; they could be mediators of host defense against the microbial challenge, or mediators for periodontal tissue alteration. Osteopontin (OPN) is a glycosylated phosphoprotein, it functions as a Th 1 (T - helper 1) cytokine, promotes cell-mediated immune responses and plays a role in chronic inflammatory diseases. To assess OPN expression in dentogingival junction (DGJ) in chronic periodontitis, ten 13-16 months old, beagle dogs were used. Animals were distributed randomly into two equal groups: Group I: animals had healthy periodontium. Group II: chronic periodontitis was induced in animals. Four weeks after induction, dogs were sacrificed and specimens were prepared for immunohistochemical staining using OPN monoclonal antibody. Intensity of OPN reactions was recorded by image J and t-test was used to compare between the two groups. Healthy periodontium showed a mild OPN reaction. While, in chronic periodontitis, moderate to intense extracellular reaction was seen in DGJ. Also, sporadic nuclear OPN reactions of inflammatory cells were noticed in chronic periodontitis. T-test revealed a significant difference between normal and chronic periodontitis groups. In conclusion, OPN may play a role in chronic inflammation and can be considered as a biomarker of periodontal disease progression.
\end{abstract}

Keywords: OPN, Chronic Periodontitis, DGJ, Periodontium, Dogs

\section{Introduction}

DGJ is a unique anatomic feature that functions to attach gingiva to the tooth. It comprises of epithelial and connective tissue portions, both of which are of fundamental importance for periodontal pathogenesis. The epithelial portion can be divided into three different structures: gingival, sulcular, and junctional epithelium. These epithelial structures are in continuity with each other, but they have distinct structures and functions ${ }^{[1]}$. Tsukamoto et al., 2012 stated that the junctional epithelium provided the front-line defense against periodontal bacterial infection. The migration of inflammatory cells into the junctional epithelium might represent a protective reaction against bacterial invasion ${ }^{[2]}$.

Periodontal diseases are chronic inflammatory disorders that affect the attachment apparatus of the teeth. They are considered to be the major cause of tooth loss in adults and the most prevalent form of alveolar bone destruction in human $^{[3]}$. Inflammatory periodontal diseases are initiated and maintained by plaque bacteria and their metabolic products, which trigger the local infiltration of inflammatory cells associated with the degradation of extracellular matrix macromolecules ${ }^{[4,5]}$. The term periodontal disease refers to both, periodontitis and gingivitis. Gingivitis is one of the most common oral diseases in adult populations worldwide. It affects about $10 \%$ to $30 \%$ of the general population, in whom, gingivitis quickly progresses to periodontitis $[4,5]$. Chronic gingival inflammation may result in gingival enlargement or hyperplasia. This overgrowth of gingiva may be exaggerated in patient with poor oral hygiene, genetic or drug-related systemic factors ${ }^{[6]}$. Lately, the critical role of cytokines in the pathogenesis of periodontal destruction had been considered. Not only as mediators of host defense, but also as mediators of periodontal tissues change ${ }^{[4,6]}$.

OPN is a glycosylated phosphoprotein found in all body fluids and in protein matrix of mineralized tissues. Although it is highly expressed in bone, it is also expressed by various cell types including macrophages, endothelial cells, smooth muscle cells and epithelial cells ${ }^{[7,8]}$. OPN is a multifunctional 
protein; it can function both; as a cell attachment protein and as a cytokine, delivering signals to cells via a number of receptors including several integrins and CD44. Expression of OPN is enhanced by a variety of toxicants, especially those that activate protein kinase $\mathrm{C}$, as it is involved in the recruitment and retention of macrophages and $\mathrm{T}$ cells to inflamed sites. It may not only be expressed by $\mathrm{T}$ cells, but also induces $\mathrm{T}$-cell chemotaxis and stimulats $\mathrm{T}$-cell proliferation ${ }^{[9-11]}$. Moreover, the expression of OPN is affected by a number of substances including hormones (e.g., vitamin D3 and estrogen), cytokines, growth factors and inflammatory mediators ${ }^{[12]}$. Many experiments have suggested that OPN may inhibit apoptosis, possibly contributing to the survival of cells in response to toxicant injury. Elevated OPN expression often correlates with malignancy and has been shown to enhance the tumorigenic and/or metastatic phenotype of cancer cells. Recent studies have revealed the critical roles of OPN in bone remodeling and cell-mediated immunity ${ }^{[10,12]}$. It is worth mentioning that OPN may exert both pro and anti inflammatory actions, with the net effect depending upon the nature of the biological scenario ${ }^{[11]}$.

Although, the role of OPN in several pathophysiological events in a variety of tissues has been studied, there are few studies that had highlighted the potential role of OPN in oral disease. Here, we will provide an insight into the possible role of OPN in inflammatory periodontal disease. So, this study was conducted to evaluate OPN expression in DGJ with chronic periodontitis using beagle dogs.

\section{Material and Methods}

Ten, 13-16 months old, beagle dogs weight approximately 10 kgs were used. Animals were obtained from the animal house, Faculty of Medicine, Tanta University. The present study was approved by Research Ethical Committee of Tanta University. Animals were housed under controlled temperature and lighting conditions, with free access to standard food and water. The experimental protocol was designed in accordance with the guidelines for the responsible use of animals in research as a part of scientific research ethics recommendations ${ }^{[13]}$. According to the methodology of previous studies, animals were distributed randomly into two equal groups: Group I (control group): Animals were not subjected to chronic periodontitis. Group II (Chronic periodontitis group): Chronic periodontitis was induced in animals. Induction of periodontitis was performed using cotton ligatures in subgingival position in the distal aspect of each mandibular premolar. It was induced under general anesthesia using intravenous injection of ketamine $(40 \mathrm{mg} / \mathrm{kg})$ [14]. After four weeks of undisturbed plaque accumulation, clinical signs of inflammation (edema, redness and bleeding on gentle propping) were evident in the periodontal tissues. All dogs were sacrificed with an overdose of Phenobarbital sodium salt ${ }^{[14]}$. Mandibles of all dogs were dissected carefully keeping the gingival attachment intact with bone. Specimens were processed for subsequent immunohistochemical analysis. They were fixed in $10 \%$ buffered formalin for 24 hours and decalcified in $10 \%$ neutral-buffered ethylenediaminetetraacetic acid (EDTA) at room temperature for three months. Afterward, they were dehydrated through ascending graded series of ethanol, and processed into paraffin. $5 \mu \mathrm{m}$ mesio-distal serial sections were cut and mounted on commercially available positively charged glass slides.

\subsection{Immunohistochemical Staining}

Immunohistochemical labelling was performed using the avidin-biotin-complex (ABC) method. Representative sections were deparaffinized in xylene and re-hydrated through a descending series of ethanol concentrations. The sections were washed with TBS $(20 \mathrm{mM}$ Tris- $\mathrm{HCl}, 150 \mathrm{mM}$ $\mathrm{NaCl}, \mathrm{pH}$ 7.4). Then they were incubated in $0.3 \% \mathrm{H}_{2} \mathrm{O}_{2}$ in $\mathrm{dH}_{2} \mathrm{O}$ at room temperature $(30 \mathrm{~min}$ ) to inhibit endogenous peroxidase. Antigen retrieval was performed according to the manufacture recommendations. Slides were placed in $100 \mu \mathrm{l}$ blocking solution (Abcam), for 30 minutes at room temperature. Rabbit polyclonal primary antibody to OPN (Cat. No. ab8448, Abcam, Cambridge, UK) was applied at recommended dilutions at $4^{\circ} \mathrm{C}$ overnight. For negative control, the primary antibody was replaced with only the blocking buffer. All sections were washed in 1X Phosphate buffered saline (PBS) and then incubated with anti rabbit secondary biotinylated antibody (in blocking buffer) for 1 hour at room temperature in a humidified chamber. To perform peroxidase visualization; sections were incubated in $\mathrm{ABC}$ solution for 1 hour at room temperature. Color reaction was then developed by adding DAB (3, 3'-diaminobenzidine) solution $\left(0.5 \mathrm{mg} / \mathrm{ml} \mathrm{DAB}\right.$ and $\left.0.1 \% \mathrm{H}_{2} \mathrm{O}\right)$ onto the sections. When color reaction was satisfactory, it was stopped by rinsing with $\mathrm{H}_{2} \mathrm{O}$ for 5-10 minutes, and then sections were counterstained with hematoxylin for 2 minutes. Sections were gradually dehydrated and mounted with cover slips.

\subsection{Evaluation of Immunohistochemical Staining}

Immunohistochemical staining was assessed using Leica light microscope. Images of the immunostaind sections were analyzed for OPN staining intensity using image $\mathbf{J}$ analysis system (ImageJ 1.48s, Wayne Rasband, National Institute of Health, USA).

\subsection{Statistical Analysis}

Independent t-test was used for statistical analyses. It was performed using GraphPad Prism software for windows (V 5.01, California, USA). All data were expressed as mean \pm standard deviation (SD). The t-test was used for statistical comparison between the two groups. A $P$-value of less than 0.05 was considered to be statistically significant.

\section{Results}

\subsection{Immunohistochemical Findings}

Group I (Normal periodontium): The DGJ of this group exhibited mild expression of OPN in both epithelium and the 
underlying connective tissue (CT). OPN reaction was distributed in the extracellular matrix of all layers of gingival epithelium except the cornified layer which had a negative reaction (Fig.1, A-B). Negative control did not show any positive staining (Fig.1, C-D).

Group II (Chronic periodontitis): Animals with induced chronic periodontitis had epithelial hyperplasia with CT chronic inflammatory cells infiltration. All epithelial components of DGJ; gingival, sulcular and junctional epithelium showed moderate extracellular OPN expression. The reaction was detected in all layers of gingival epithelium except the cornified layer which had a negative reaction. The underlying CT exhibited intense OPN reaction. Some of chronic inflammatory cells exhibited intense nuclear OPN reaction. Moreover the endothelial cells lining the blood vessels exhibited moderate reaction (Fig. 2).

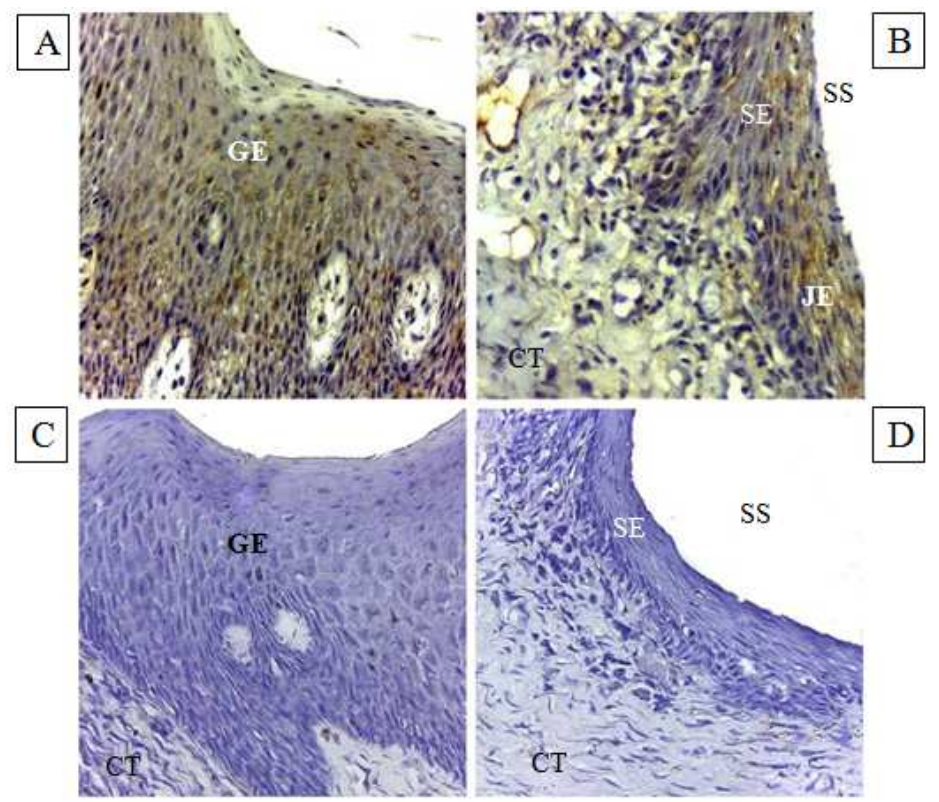

Figure 1. Immunolocalization of mild OPN expression in dog healthy DGJ. (A) Extracellular OPN expression is evident in all layers of gingival epithelium except the cornfield layer, has a negative reaction. (B) Shows OPN expression in sulcular epithelium, junctional epithelium and underlying CT. (C-D) Negative control did not show any positive staining. Sulcular epithelium (SE), Gingival epithelium (GE), Sulcular space (SS), Junctional epithelium (JE), Connective tissue (CT). (DAB, A- C $\times 40 \& D \times 10)$.
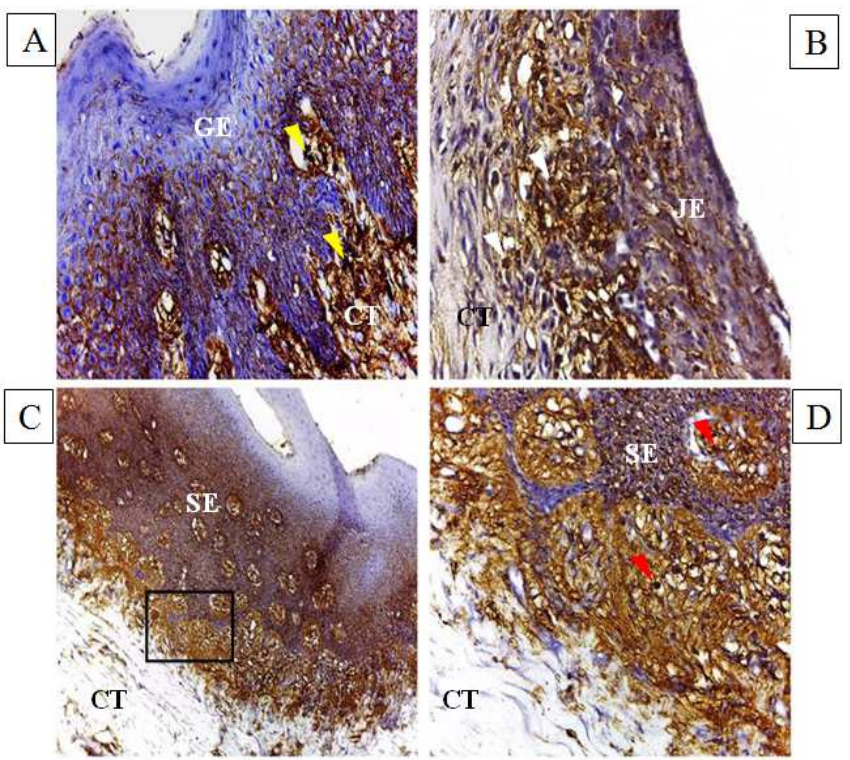

Figure 2. Immunolocalization of moderate to intense OPN expression in dog DGJ with chronic periodontitis. (A) Shows extracellular expression in gingival epithelium and connective tissue (Yellow arrowheads). However, the cornified layer has a negative reaction. (B) Shows extracellular OPN expression in junctional epithelium. The underlying CT exhibits intense extracellular reaction. Some of chronic inflammatory cells show intense nuclear OPN reaction (White arrowheads). (C) Shows extracellular OPN expression in sulcular epithelium and the underlying CT. (D) High magnification of the boxed area in (C) showing epithelial OPN moderate expression, whereas the underlying CT has an intense reaction. Some of chronic inflammatory cells exhibit intense nuclear OPN reaction (Red arrowheads). Gingival epithelium (GE), Sulcular epithelium (SE), Junctional epithelium (JE). Connective tissue (CT). (DAB, A, B and D $x$ $40, C \times 10)$. 


\subsection{Histomorphometric and Statistical Analysis}

Independent t-test was used to compare the mean intensity of OPN expression between control and chronic periodontitis groups. In normal periodontium, the mean OPN expression intensity was $3.22 \pm 0.079$ and this was found to be significantly low when compared to that of the chronic periodontitis group 29.60 $\pm 7.39, p=0.006$ (Fig.3).

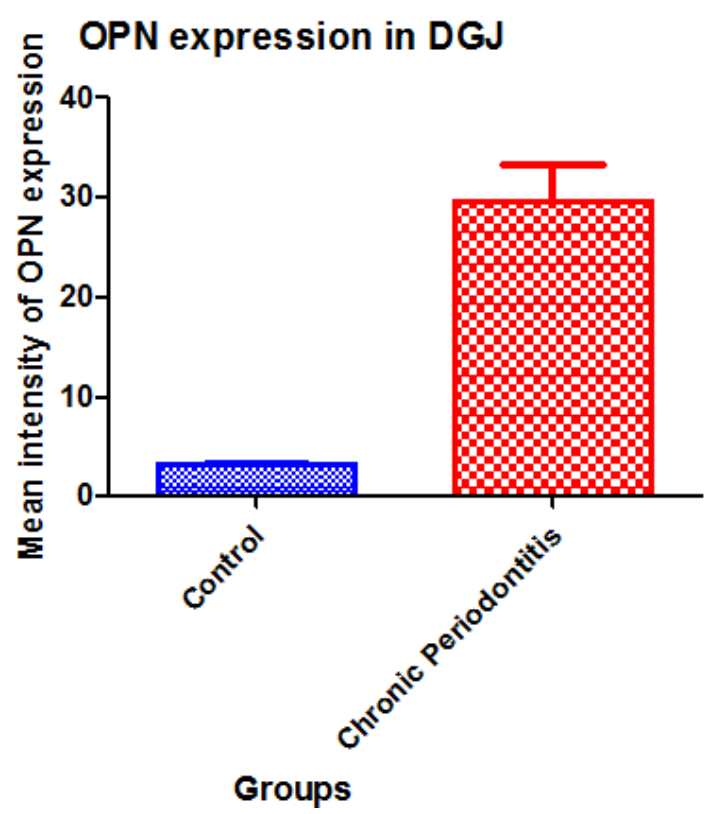

Figure 3. Mean intensity of $O P N$ expression in control and chronic periodontitis groups. OPN mean intensity was significantly higher in chronic periodontitis group when compared with the control one, $P=0.006$

\section{Discussion}

Periodontal disease is either acute or chronic inflammatory condition, which develops as a result of imbalance between the host defense and microbial agents. This results in toothsupporting tissues destruction leading to tooth mobility and eventually tooth loss. However, at the molecular and cellular levels, the inflammatory process is defined by cellular infiltrates and the release of a variety of cytokines ${ }^{[15]}$. The main provoking factor that induces inflammation of gingival tissue is the presence of bacterial biofilm (dental plaque) on the teeth/gingival interfaces. The products of which such as lipopolysaccharide (LPS) molecules, are known to initiate a chain of reactions in the tissue leading to host response as well as destructive process ${ }^{[16,17]}$.

OPN is defined as a highly modified integrin-binding extracellular matrix glycophosphoprotein produced by cells of the immune system, epithelial tissue, smooth muscle cells, osteoblasts, and tumor cells ${ }^{[18]}$. OPN was first identified as a bone matrix protein with an adhesive function owing to its integrin binding activity. Later on, many studies have been reported that OPN was expressed in various tissues and was up regulated under pathological as well as physiological conditions ${ }^{[19,20]}$. Extensive researches had elucidated the pivotal role of OPN in cell signaling that controls inflammation, tumor progression, and metastasis. OPN interaction with the integrin receptors expressed by inflammatory cells through its arginine glycine-aspartate (RGD) and non-RGD motifs promote migration and adhesion of cells ${ }^{[18]}$.

In this study we highlighted the expression of OPN by cells of gingiva in normal and inflammatory conditions using induced chronic periodontitis in dogs as an experimental model.

In control group, mild OPN extracellular expression in DGJ was noticed. The expression of OPN in normal tissue elucidated its broad range of biological functions. Several studies reported that OPN was widely distributed in the body and had been implicated in several physiological functions including cell adhesion, migration, signaling, inflammation, and biomineralization ${ }^{[21-23]}$.

In current study we used cotton ligature in subgingival area around the neck of dogs premolar teeth to induce chronic periodontitis. This method was in agreement with Kim et al., 2012 and Gab Allah et al., 2014 who found that, four weeks after ligature, there was severe destruction of DGJ, periodontal tissues and resorption of both; root and alveolar bone. In addition, gingival inflammation and apical migration of attachment epithelium along the affected root surface were reported ${ }^{[24,25]}$. The DGJ of dogs with induced chronic periodontitis revealed intense OPN extra cellular expression by epithelial cells of hyperplastic gingival layers except the cornfield one which had a negative reaction. Also, CT had both nuclear and extracellular expressions. In addition, chronic inflammatory cells exhibited intense nuclear reaction while endothelial cells of blood vessels exhibited a moderate one. These results had been explained by many researchers who had focused on the roles of OPN in the pathogenesis of various diseases and its pathological roles as a pro-inflammatory and pro-fibrotic cytokine ${ }^{[19,20]}$. The increased expression of OPN in hyperplastic epithelium was in agreement with Takahashi et al., 2001 and Pardo et al., 2005 who stated that, OPN was a fibrogenic cytokine and its expression was elevated in hyperplastic alveolar epithelial cell (AEC) type II of human idiopathic pulmonary fibrosis (IPF) and murine bleomycininduced lung fibrosis $[19,20]$. Furthermore, the increased expression of OPN during inflammation and many pathological conditions was affected by a number of substances including hormones, cytokines, and growth factors. Several inflammatory mediators and growth factors such as interleukin- 1 (IL-1), tumor necrosis factor alpha, and plateletderived growth factor are known to stimulate OPN transcription via activation of protein kinase $\mathrm{C}^{[12]}$

It is worth mentioning that OPN regulates the immune system at many different levels. It serves as a chemotactic molecule to promote the migration of inflammatory cells to the wound site. Also, it acts as an adhesive protein to retain cells at the site. In addition, OPN functions as a pro-inflammatory cytokine and can modulate the immune response by enhancing expression of Th1 cytokines and matrix degrading enzymes ${ }^{[22,}$ 
23]. OPN plays a pivotal role in $\mathrm{T}$ cell and macrophage responses during cell mediated immune responses against bacterial and viral pathogens ${ }^{[26]}$. Moreover, it has been shown to modulate dendritic cell responses and neutrophil chemotaxis at the inflammation site ${ }^{[26,27]}$. The current results suggested that OPN might act as a regulator for promoting and migration of inflammatory cells at the site of chronic inflammation. This suggestion was explained by Tsai et al., 2005 who reported that, macrophages were both; source and target of OPN. Furthermore, they had also shown that OPN regulated foreign body giant cell (FBGC) formation in vitro and in vivo ${ }^{[28]}$.

Statistical analysis revealed significant difference in OPN expression between the two groups. The expression of OPN was significantly increased at chronic periodontitis group when compared to the control one. This is in agreement with Sharma and Pradeep, 2006 who concluded that, OPN levels increased in gingival crevicular fluid (GCF) as it progressed from healthy to periodontitis stage. Interestingly, periodontal treatment resulted in the reduction of its levels ${ }^{[29]}$.

In conclusion, the data in this study stated that, OPN was expressed in normal DGJ. However, its expression was increased with chronic periodontitis. These findings suggest that OPN may play a key role in chronic inflammation, and can be considered as a biomarker of periodontal disease progression.

\section{Acknowledgment}

This work was financially supported by the scientific research administration, Tanta University through the program of research projects fund in the field of biotechnology. Also, authors would like to thank Dr. Heba Mohammed El Tokhey, professor of oral biology department, faculty of dentistry, Tanta University, for her invaluable support.

\section{References}

[1] Nanci, A. and D.D. Bosshardt, Structure of periodontal tissues in health and disease. Periodontol 2000, 2006. 40: p. 11-28.

[2] Tsukamoto, Y., M. Usui, G. Yamamoto, Y. Takagi, T. Tachikawa, M. Yamamoto, and M. Nakamura, Role of the junctional epithelium in periodontal innate defense and homeostasis. J Periodontal Res, 2012. 47(6): p. 750-7.

[3] Savage, A., K.A. Eaton, D.R. Moles, and I. Needleman, A systematic review of definitions of periodontitis and methods that have been used to identify this disease. J Clin Periodontol, 2009. 36(6): p. 458-67.

[4] Grigoriadou, M.E., S.O. Koutayas, P.N. Madianos, and J.R. Strub, Interleukin-1 as a genetic marker for periodontitis: review of the literature. Quintessence Int, 2010. 41(6): p. 51725 .

[5] Seguier, S., G. Godeau, and N. Brousse, Collagen fibers and inflammatory cells in healthy and diseased human gingival tissues: a comparative and quantitative study by immunohistochemistry and automated image analysis. J Periodontol, 2000. 71(7): p. 1079-85.
[6] Seguier, S., G. Godeau, and N. Brousse, Immunohistological and morphometric analysis of intra-epithelial lymphocytes and Langerhans cells in healthy and diseased human gingival tissues. Arch Oral Biol, 2000. 45(6): p. 441-52.

[7] O'Brien, E.R., M.R. Garvin, D.K. Stewart, T. Hinohara, J.B. Simpson, S.M. Schwartz, and C.M. Giachelli, Osteopontin is synthesized by macrophage, smooth muscle, and endothelial cells in primary and restenotic human coronary atherosclerotic plaques. Arterioscler Thromb, 1994. 14(10): p. 1648-56.

[8] Thorat, M., P. Ar, and G. Garg, Correlation of Levels of Oncostatin M Cytokine in Crevicular Fluid and Serum in Periodontal Disease. International Journal of Oral Science, 2010. 2(4): p. 198-207.

[9] Malyankar, U.M., M. Almeida, R.J. Johnson, R.H. Pichler, and C.M. Giachelli, Osteopontin regulation in cultured rat renal epithelial cells. Kidney Int, 1997. 51(6): p. 1766-73.

[10] Devoll, R.E., W. Li, K.V. Woods, G.J. Pinero, W.T. Butler, M.C. Farach-Carson, and R.P. Happonen, Osteopontin (OPN) distribution in premalignant and malignant lesions of oral epithelium and expression in cell lines derived from squamous cell carcinoma of the oral cavity. J Oral Pathol Med, 1999. 28(3): p. 97-101.

[11] Denhardt, D.T., C.M. Giachelli, and S.R. Rittling, Role of osteopontin in cellular signaling and toxicant injury. Annu Rev Pharmacol Toxicol, 2001. 41: p. 723-49.

[12] Denhardt, D.T. and M. Noda, Osteopontin expression and function: role in bone remodeling. J Cell Biochem Suppl, 1998. 30-31: p. 92-102.

[13] Knight, A., ed. The Costs and Benefits of Animal Experiments. May 2011, Palgrave Macmillan.272.

[14] Nociti, F.H., Jr., R. Cesco De Toledo, M.A. Machado, C.M. Stefani, S.R. Line, and R.B. Goncalves, Clinical and microbiological evaluation of ligature-induced peri-implantitis and periodontitis in dogs. Clin Oral Implants Res, 2001. 12(4): p. 295-300.

[15] Patarca, R., G.J. Freeman, R.P. Singh, F.Y. Wei, T. Durfee, F. Blattner, D.C. Regnier, C.A. Kozak, B.A. Mock, H.C. Morse, 3rd, and et al., Structural and functional studies of the early $\mathrm{T}$ lymphocyte activation 1 (Eta-1) gene. Definition of a novel T cell-dependent response associated with genetic resistance to bacterial infection. J Exp Med, 1989. 170(1): p. 145-61.

[16] Slots, J. and M.A. Listgarten, Bacteroides gingivalis, Bacteroides intermedius and Actinobacillus actinomycetemcomitans in human periodontal diseases. J Clin Periodontol, 1988. 15(2): p. 85-93.

[17] Socransky, S.S., A.D. Haffajee, J.L. Dzink, and J.D. Hillman, Associations between microbial species in subgingival plaque samples. Oral Microbiology and Immunology, 1988. 3(1): p. $1-7$.

[18] Ramaiah, S.K. and S. Rittling, Pathophysiological role of osteopontin in hepatic inflammation, toxicity, and cancer. Toxicol Sci, 2008. 103(1): p. 4-13.

[19] Takahashi, F., K. Takahashi, T. Okazaki, K. Maeda, H. Ienaga, M. Maeda, S. Kon, T. Uede, and Y. Fukuchi, Role of osteopontin in the pathogenesis of bleomycin-induced pulmonary fibrosis. Am J Respir Cell Mol Biol, 2001. 24(3): p. 264-71. 
[20] Xie, Y., M. Sakatsume, S. Nishi, I. Narita, M. Arakawa, and F. Gejyo, Expression, roles, receptors, and regulation of osteopontin in the kidney. Kidney Int, 2001. 60(5): p. 1645-57.

[21] Weber, G.F., S. Zawaideh, S. Hikita, V.A. Kumar, H. Cantor, and S. Ashkar, Phosphorylation-dependent interaction of osteopontin with its receptors regulates macrophage migration and activation. J Leukoc Biol, 2002. 72(4): p. 752-61.

[22] Pardo, A., K. Gibson, J. Cisneros, T.J. Richards, Y. Yang, C. Becerril, S. Yousem, I. Herrera, V. Ruiz, M. Selman, and N. Kaminski, Up-regulation and profibrotic role of osteopontin in human idiopathic pulmonary fibrosis. PLoS Med, 2005. 2(9): p. e251.

[23] Bruemmer, D., A.R. Collins, G. Noh, W. Wang, M. Territo, S. Arias-Magallona, M.C. Fishbein, F. Blaschke, U. Kintscher, K Graf, R.E. Law, and W.A. Hsueh, Angiotensin II-accelerated atherosclerosis and aneurysm formation is attenuated in osteopontin-deficient mice. J Clin Invest, 2003. 112(9): p. 1318-31.

[24] Olfat M. Gab Allah, H.M.E.T., Mona A. Abd-Elmotelb, Hoda M. El-Guindy, Reda G. Saleh, Nanotechnology and its Role in the Treatment of Induced Periodontitis (Experimental Study). rnal of American Scienc, 2014. 10(10): p. 179-195.
[25] Kim, S.E., E.R. Lee, Y. Lee, M. Jeong, Y.W. Park, J.S. Ahn, J.T. Ahn, and K. Seo, A modified method for inducing periodontitis in dogs using a silk-wire twisted ligature. Journal of Veterinary Science, 2012. 13(2): p. 193-197.

[26] Ashkar, S., G.F. Weber, V. Panoutsakopoulou, M.E. Sanchirico, M. Jansson, S. Zawaideh, S.R. Rittling, D.T. Denhardt, M.J. Glimcher, and H. Cantor, Eta-1 (osteopontin): an early component of type-1 (cell-mediated) immunity. Science, 2000. 287(5454): p. 860-4.

[27] Yu, X.Q., D.J. Nikolic-Paterson, W. Mu, C.M. Giachelli, R.C. Atkins, R.J. Johnson, and H.Y. Lan, A functional role for osteopontin in experimental crescentic glomerulonephritis in the rat. Proc Assoc Am Physicians, 1998. 110(1): p. 50-64.

[28] Tsai, A.T., J. Rice, M. Scatena, L. Liaw, B.D. Ratner, and C.M. Giachelli, The role of osteopontin in foreign body giant cell formation. Biomaterials, 2005. 26(29): p. 5835-43.

[29] Sharma, C.G. and A.R. Pradeep, Plasma and crevicular fluid osteopontin levels in periodontal health and disease. J Periodontal Res, 2007. 42(5): p. 450-5. 